\title{
Hatnoub (2019)
}

Yannis Gourdon, Roland Enmarch, Olivier Lavigne, Jérôme Fage, Dominique Farout, Sameh Michel, Maël Crépy, Thomas Sagory et Benoît Touchard

\section{OpenEdition \\ Journals}

Édition électronique

URL : https://journals.openedition.org/baefe/1028

DOI : $10.4000 /$ baefe. 1028

ISSN : 2732-687X

Éditeur

ResEFE

\section{Référence électronique}

Yannis Gourdon, Roland Enmarch, Olivier Lavigne, Jérôme Fage, Dominique Farout, Sameh Michel, Maël Crépy, Thomas Sagory et Benoît Touchard, « Hatnoub (2019) » [notice archéologique], Bulletin archéologique des Écoles françaises à l'étranger [En ligne], Égypte, mis en ligne le 05 octobre 2020, consulté le 28 juin 2022. URL : http://journals.openedition.org/baefe/1028 ; DOI : https://doi.org/ $10.4000 /$ baefe. 1028

Ce document a été généré automatiquement le 11 décembre 2020.

\section{cc) $(1)$}

Le Bulletin archéologique des Écoles françaises à l'étranger est mise à disposition selon les termes de la Licence Creative Commons Attribution - Pas d'Utilisation Commerciale - Pas de Modification 4.0 International. 


\title{
Hatnoub
}

\author{
Yannis Gourdon, Roland Enmarch, Olivier Lavigne, Jérôme Fage, \\ Dominique Farout, Sameh Michel, Maël Crépy, Thomas Sagory et Benoît \\ Touchard
}

\section{NOTE DE L'AUTEUR}

Année de la campagne : 2019 (5 septembre - 4 octobre)

Numéro et intitulé de l'opération de terrain : 17134 - Les carrières de travertin égyptien de Hatnoub

Composition de l'équipe de terrain : L'équipe était composée de Yannis Gourdon (égyptologue, université Lumière Lyon 2), Roland Enmarch (égyptologue, University of Liverpool), Olivier Lavigne (tailleur de pierre), Dominique Farout (égyptologue, École du Louvre, Institut Khéops), Jérôme Fage (archéologue, musée de Saint-Romain-en-Gal), Thomas Sagory (photographe, ministère de la Culture), Benoît Touchard (photographe, Summum 3D), Vincent Morel (doctorant en égyptologie, université de Genève), Maël Crépy (géomorphologue, CNRS, UMR 5189 HiSoMA), Anita Quiles (responsable du laboratoire d'archéométrie, Ifao), Hassân El-Amir (conservateur-restaurateur, Ifao) et Younis Ahmed Mohamadein (conservateur-restaurateur, Ifao). L'intendance et le soutien aux opérations de terrain ont été assurés par Sameh Michel. Le ministère des Antiquités était représenté par Mohammed Khalil Mohammed Khalil.

Partenariats institutionnels : Cette mission conjointe de l'Ifao et de l'université de Liverpool est menée avec le soutien technique et financier du fonds Khéops pour l'archéologie, de l'Heritage Innovation Preservation Institute et de l'Egypt Exploration Society (EES).

Les travaux de cette saison ont suivi 5 objectifs :

1. le dégagement de la terrasse qui surplombe la partie basse de la paroi nord de la descenderie et la paroi nord-ouest du cirque de la carrière $P$;

2. la poursuite du dégagement du dispositif de halage des blocs mis au jour à la toute fin de la saison 2018, dans le sondage transversal dans le bas de la descenderie de la carrière P ; 
3. le dégagement d'un grand bâtiment (SW 1) non répertorié repéré l'an passé au sud-ouest de la carrière $P$;

4. la poursuite du travail sur les inscriptions et notamment leur restauration;

5. la mise en œuvre du survey géomorphologique des environs de la carrière $\mathrm{P}$, en lien avec la poursuite du survey archéologique du plateau.

2 En marge des opérations scientifiques, la mission a accueilli une équipe de tournage de la $\mathrm{BBC}$ pour la réalisation d'un documentaire.

\section{Les travaux dans la carrière $P$}

\subsection{Le dégagement de la voie de halage}

Yannis Gourdon

Le dégagement de la voie de halage entrepris l'an passé dans la partie basse de la descenderie a été poursuivi cette année par Y. Gourdon et R. Enmarch, assistés de S. Michel. Toute la largeur de la voie avec la rampe centrale et ses deux volées d'escalier a ainsi été dégagée. Dans ce secteur, les marches sont bien moins préservées que dans la partie haute de la descenderie et une grande fissure est apparente au niveau du sol du côté de la paroi sud. Les installations liées au système de halage visibles en partie basse de la descenderie comprennent aussi de chaque côté un trou de poteau carré, dont un (paroi nord) encore muni d'une pierre de calage en place, et une ébauche de trou de poteau carré. Des traces au sol pouvant indiquer la présence d'emplacements de traverses ont également été repérées. Les éléments du système de halage dégagé à ce jour semblent bien former un ensemble cohérent qui pourrait se développer en pente constante de l'ordre de $29 \%$ sur l'intégralité de la descenderie.

Fig. 1. Vue du système de halage au fond du sondage transversal dans la partie basse de la descenderie de la carrière $P(Y$. Gourdon).

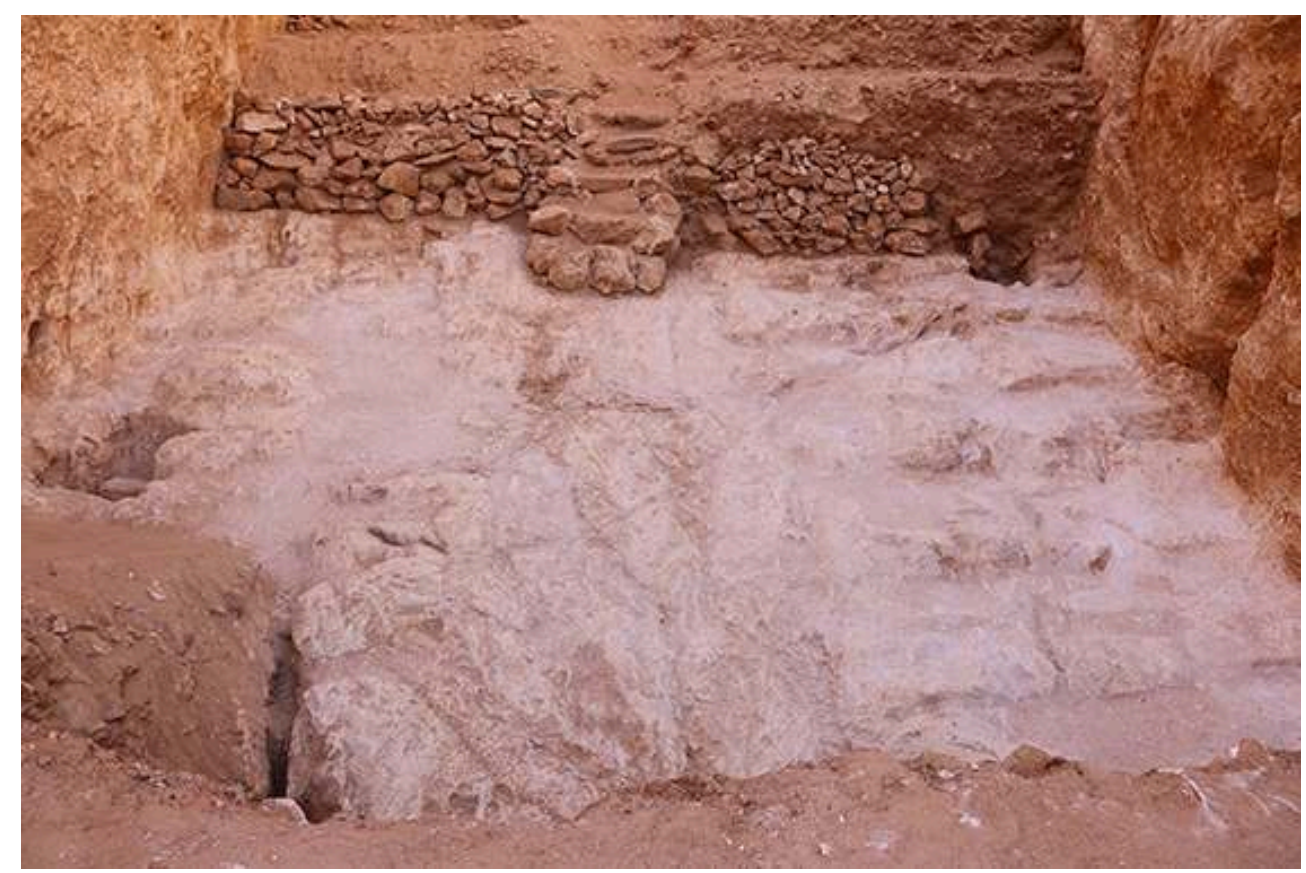

(C) Ifao. 17134_2019_NDMPF_001 
On notera enfin la découverte dans les gravats d'une ébauche de statuette en albâtre calcite de mauvaise qualité, représentant une figure humaine ou simiesque.

Fig. 2. Ébauche de la statuette en albâtre calcite découverte dans la carrière $P$ (B. Touchard).

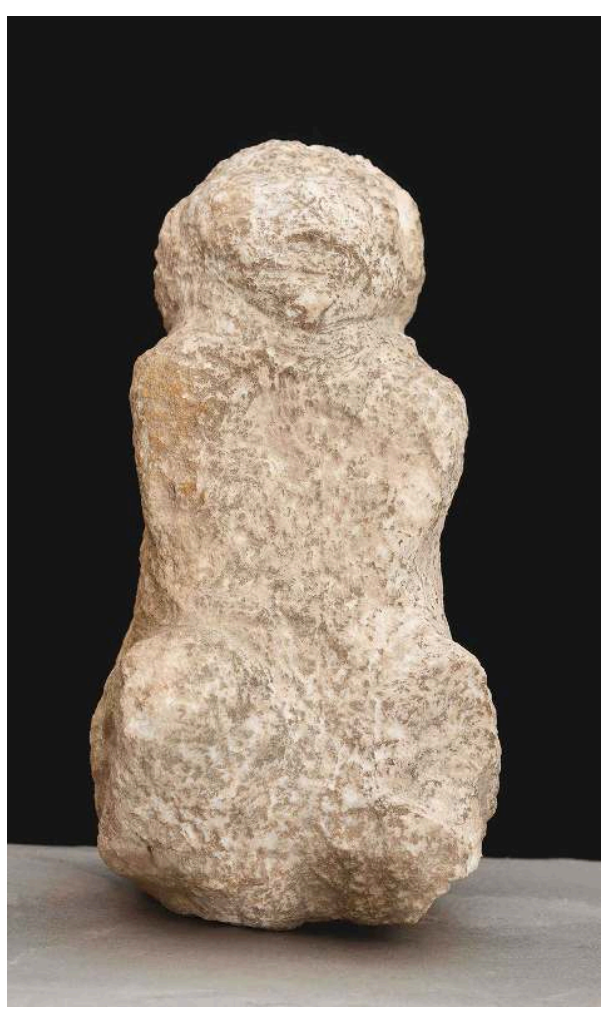

(C) Ifao. 17134_2019_NDMPM_001

\subsection{Le dégagement d'une terrasse supérieure}

Yannis Gourdon, Olivier Lavigne

Les éléments les plus intéressants, cette année, sont venus de la terrasse qui surplombe la partie basse de la paroi nord de la descenderie et la paroi nord-ouest du cirque. Déjà repérée les années précédentes, elle semblait avoir fait l'objet d'un aménagement spécifique. Le dégagement de ce secteur, entrepris en tout début de mission par Y. Gourdon, R. Enmarch et $\mathrm{O}$. Lavigne, a confirmé la présence d'un passage étroit taillé dans la roche permettant d'accéder depuis le sommet de la carrière à un escalier assez raide aux marches grossièrement taillées débouchant sur ladite terrasse. Son sol, peu horizontal, se situe en face d'une encoche visible dans la partie supérieure de la paroi sud. Ceci est à mettre en rapport avec les encoches de la paroi nord qui remontait à contre-sens de la voie de halage. Il semble bien que nous ayons à faire à un système d'échafaudage constitué d'un escalier, ou plan incliné, montant à la terrasse et d'une passerelle transversale à la voie. Ce système est visiblement doublé d'un autre un peu plus haut dans la voie. Nous avons en effet trouvé un nouveau trou de poteau qui n'a rien de commun avec les autres: il n'est pas creusé dans les marches mais sur une excroissance rocheuse de la paroi nord et ne possède pas du tout les mêmes dimensions. Nous avons également observé une encoche à l'aplomb de ce trou de poteau et une autre en face (sur la paroi sud) au même niveau. Ceci est certainement à 
mettre en relation avec les encoches visibles sur la paroi nord et elles aussi à contresens de la voie. Ici aussi il semble bien que nous ayons le même type d'installation qu'au niveau de la terrasse supérieure. Nous devrons approfondir nos recherches dans ce sens afin de définir plus précisément ces installations et leurs fonctions.

Fig. 3. Vue de la terrasse aménagée au-dessus de la partie basse de la paroi nord de la descenderie et de la paroi nord-ouest du cirque ( 0 . Lavigne).

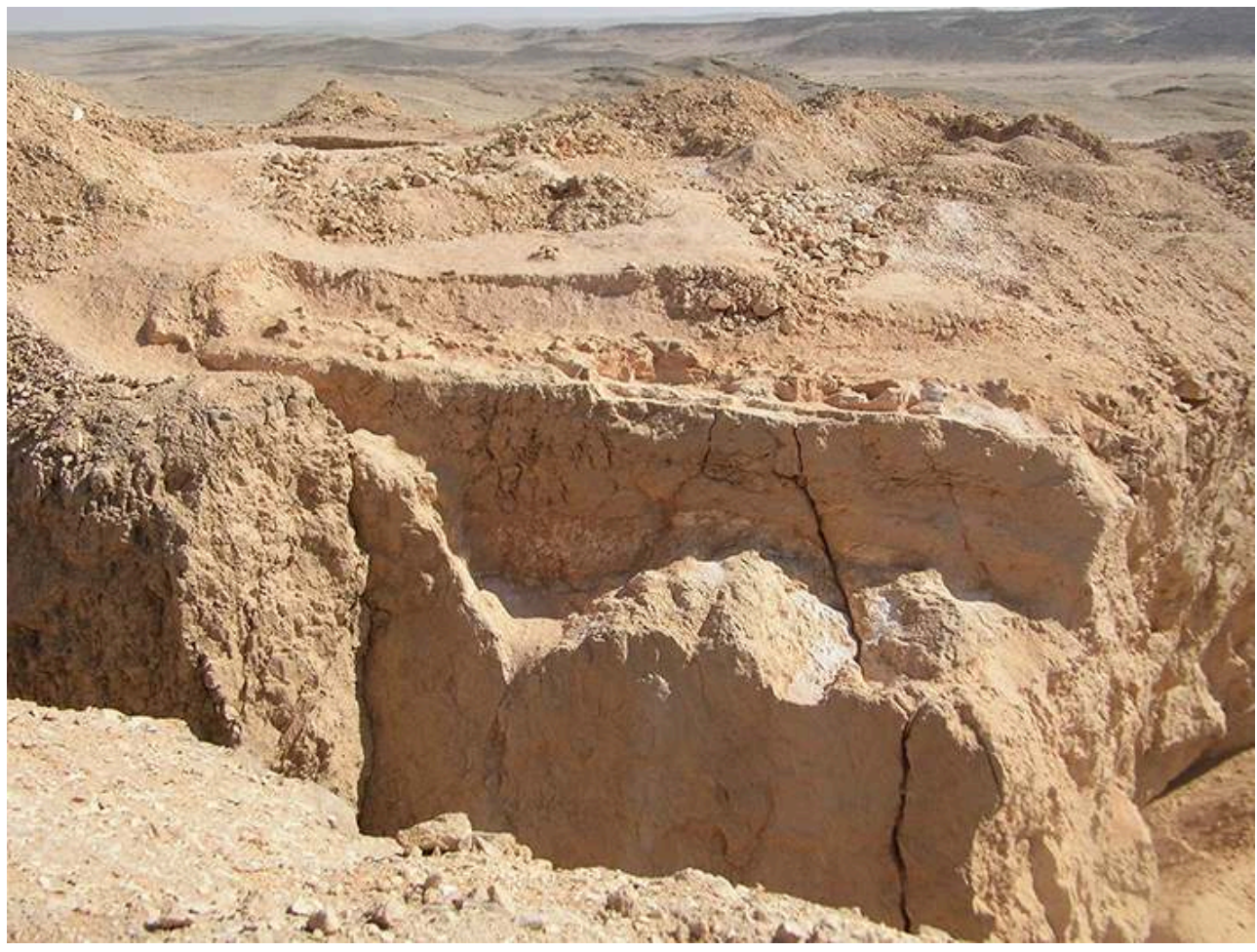

(c) Ifao. 17134_2019_NDMPF_002

\subsection{L'étude de l'outillage lithique}

Olivier Lavigne

Parallèlement à ces travaux de terrain, O. Lavigne a complété son étude des outils utilisés dans la carrière, grâce à une nouvelle collecte en surface, qui porte à présent leur nombre à 448. Le classement préliminaire a donc été repris en intégrant de nouveaux paramètres, forme générale, ergonomie, détails particuliers mais surtout traces d'usure. 0 . Lavigne a ainsi pu mettre en évidence cinq grandes familles d'outils avec leurs variantes.

Le " pic ", avec généralement son encoche pour le passage du pouce et l'adouci pour les autres doigts, possède trois variantes, celui à deux parties actives, celui avec une " tête " plate qui permet de l'utiliser en percussion posée et enfin le pic sans encoche qui est la version la plus rustique. Le "pilon», en forme de poire avec souvent une gorge en partie supérieure, se manipule à une ou deux mains, suivant sa taille (les bisphères sont de cette famille mais à l'état neuf). Les " poinçons » en forme de grosse goutte ont deux variantes, ceux simplement utilisés en percussion lancée à une ou deux mains, et ceux à percussion posée. Les outils de formes rectangulaires, dont aucun n'est neuf, appartiennent souvent à d'autres types d'outils très usés à percussion lancée et 
deux parties actives. Enfin, les "percuteurs " se décomposent eux-mêmes en deux grands sous-groupes. Les percuteurs à deux mains avec ou sans gorge sont des outils lourds utilisés pour l'extraction ou le dégrossi. Les petits percuteurs à une main sont employés pour les travaux de semi-finition, juste avant de passer au polissage, ou sont utilisés comme percuteurs pour les outils à percussion posée.

\section{Dégagement du bâtiment SW 1}

Jérôme Fage

8 dense settlement " lors de ses campagnes de prospection au sud-ouest de la carrière $\mathrm{P}$ entre les années 1985 et 1991. En 2018, la mission Hatnoub a repéré, en bordure ouest du secteur, la coupe d'un mur double présentant un appareillage soigné. révéler les vestiges d'un vaste bâtiment de plan carré de $10 \mathrm{~m}$ de côté environ, au contact direct d'une grande section en coude d'un mur d'enceinte monumental. Les objectifs de l'opération étaient de dresser le plan du bâtiment, de mieux comprendre son organisation interne et de tenter d'établir une chronologie relative de son occupation.

10 À ce stade, il nous est encore impossible de déterminer avec précision la fonction de ce bâtiment. L'absence de matériel déterminant ne facilite guère l'identification des espaces intérieurs. En revanche, la qualité remarquable de ses maçonneries et sa localisation privilégiée (à proximité de la carrière) laissent à penser que sa construction relevait d'une intention ostentatoire à établir le pouvoir royal dans le paysage.

11 Les opérations se sont réparties sur 5 secteurs :

Le secteur I concerne une enceinte monumentale dessinant deux longues sections coudées à angle droit. La structure présente un large mur parementé en moellons équarris et calibrés, doublé par un mur d'épaulement extérieur dont le parement unique présente un léger fruit en façade. L'enceinte a pu être dégagée sur près de $16 \mathrm{~m}$ linéaires et sa largeur (mur double) offre une valeur constante de 1,65 m. L'état de conservation de son élévation est particulièrement remarquable. L'enceinte est dépourvue de fondations et repose, tout comme le reste du bâtiment, sur un vaste remblai anthropique. 
Fig. 4. Vue de l'enceinte du bâtiment SW 1 (Y. Gourdon).

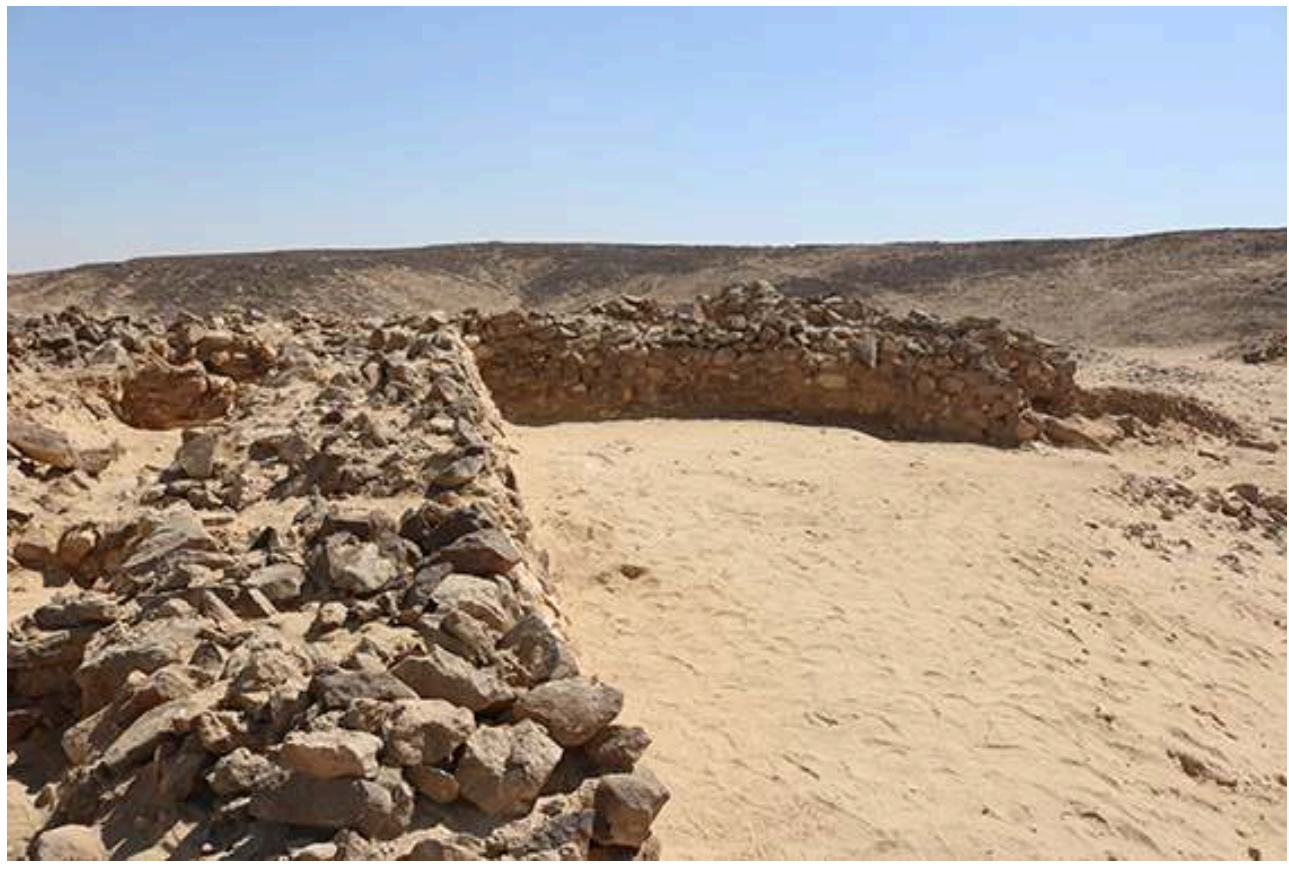

(c) Ifao. 17134_2019_NDMPF_003

12 Le secteur II couvre les abords de la façade ouest du bâtiment. Le doublage du mur disparaît au profit d'une façade soignée et parementée percée d'une porte d'accès en son centre. Le sol extérieur en connexion avec le bâtiment et le seuil sont constitués d'un remblai artificiel nivelé en terrasse.

Fig. 5. Vue de la façade ouest du bâtiment SW 1 (Y. Gourdon).

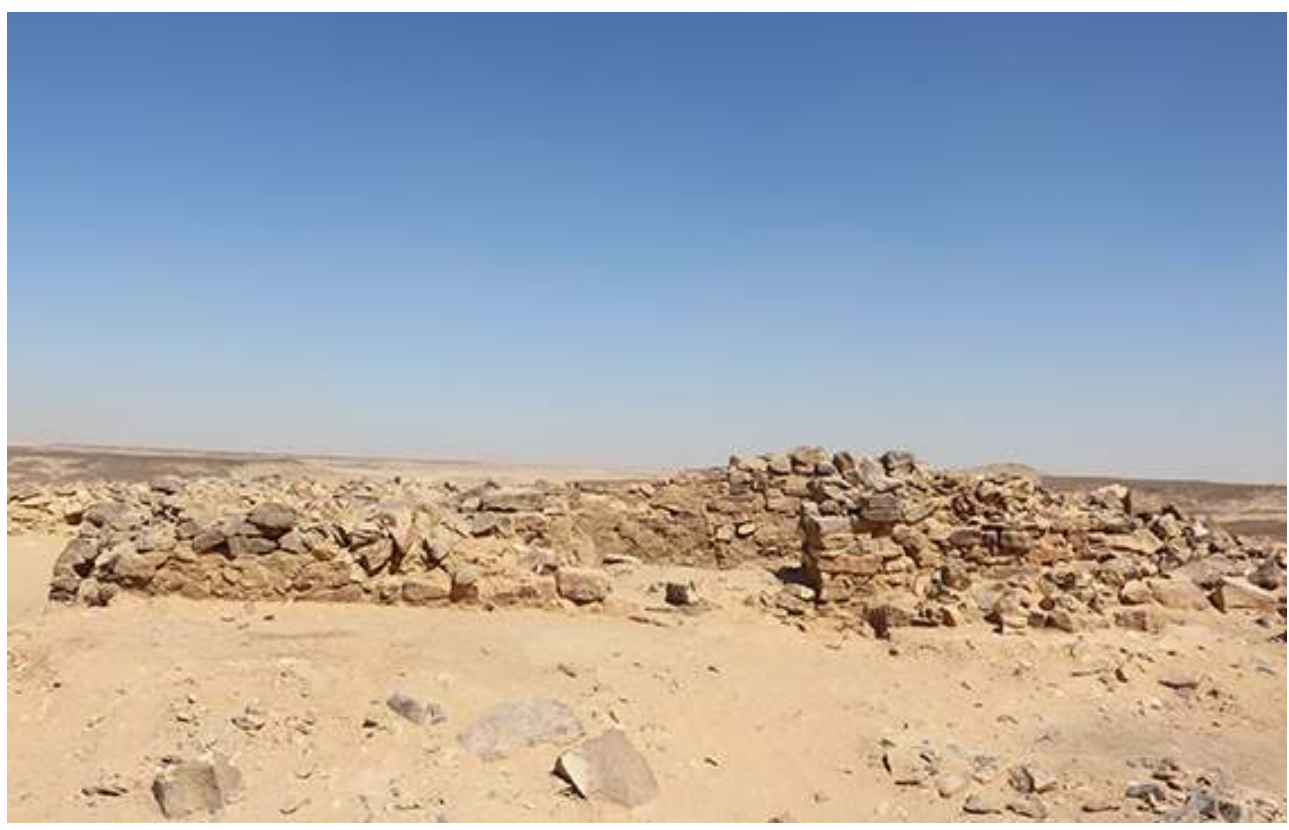

(C) Ifao. 17134_2019_NDMPF_004

13 Le secteur III, au sud du bâtiment, a révélé, malgré une importante concentration d'éboulis de blocs et d'installations postérieures (hutte, ateliers de taille), un massif 
plein de plan carré accolé au mur sud du bâtiment. Sa fonction de contrefort reste la plus plausible du fait de la déclivité forte du terrain naturel induit par le voisinage d'un ouadi.

Le secteur IV concerne l'intérieur du bâtiment de plan carré. Son dégagement complet a permis de mieux comprendre l'organisation des pièces et les logiques de circulation, et d'établir une chronologie relative. Au moins deux accès (un principal - à vestibule - à l'ouest et un secondaire au sud) sont attestés. L'étude des murs a permis d'apprécier le soin particulier apporté à la qualité des ouvrages. Des réaménagements structurels (prolongement de murs, cloisonnements, subdivisions) témoignent d'un état postérieur qui ne bouleverse pas pour autant l'orthogonalité et l'ordonnancement général du projet initial. Ces réaménagements laissent à penser que le bâtiment évolue de 4 à 6 pièces, plus un réduit très étroit localisé dans l'angle sud-ouest qui aurait pu accueillir un four à pain (structure de brique et fragments de moules à pain). Certaines des pièces accueillent par ailleurs des embellissements et améliorations de surfaces notoires (sol en mouna, enduits de torchis pariétaux lissés). Un niveau de sol commun (composition et altimétrie) est attesté sur deux tiers de la superficie du bâtiment et phasé aux structures bâties associées. Ainsi, même si le bâtiment a subi diverses modifications au cours d'une longue période d'utilisation et d'aménagements tardifs, il est à noter que leur réalisation ne bouleverse pas l'harmonie du projet original. Il s'en dégage une organisation très cohérente et réfléchie des espaces de vie (ou d'activités) et leur persistance dans le temps.

Fig. 6. Vue de l'intérieur du bâtiment SW 1 (Y. Gourdon).

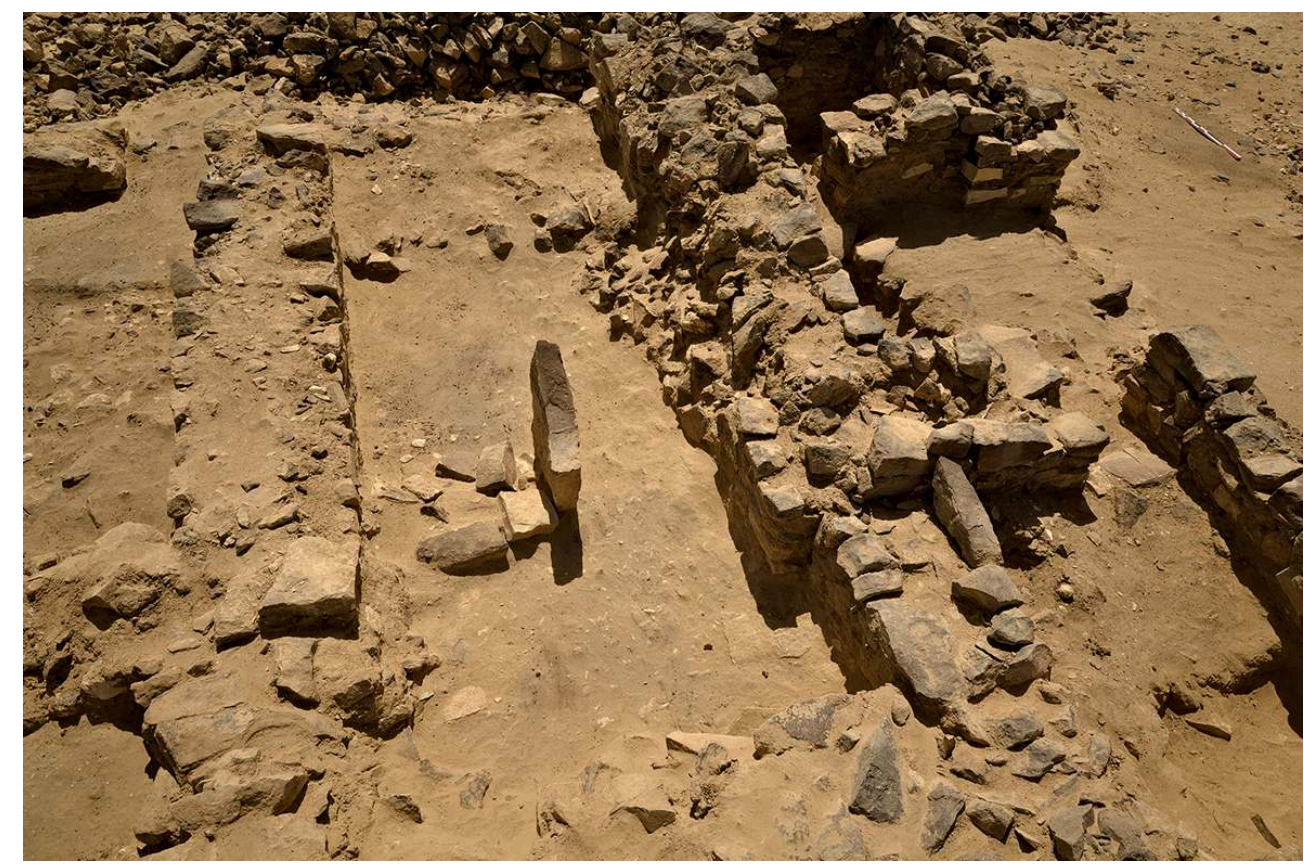

(C) Ifao. 17134_2019_NDMPF_005

Le secteur V concerne la zone située à l'est de l'enceinte nord-sud, au nord-est de SW 1 . L'objectif du dégagement était de repérer les structures en liaison avec le parement intérieur est de l'enceinte et de tenter de fixer sa terminaison nord et son hypothétique inflexion vers l'est. Trois murs ont été mis au jour (dont un percé d'une porte), tous en connexion directe avec le parement est du mur d'enceinte. Deux murs présentent une 
largeur similaire (0,70 m), tandis qu'un autre (au sud) est bien plus large (plus de $1 \mathrm{~m}$ ). $\mathrm{Au}$ nord, la terminaison de l'enceinte semble avoir été identifiée après l'enlèvement d'une première chape instable d'effondrement de blocs. Un angle intérieur est clairement visible, parfaitement chaîné au massif de maçonnerie de l'enceinte et perpendiculaire à cette dernière, en direction de l'est. Il pourrait s'agir de l'angle nord de l'enceinte, dessinant ainsi la limite septentrionale du complexe, situé à $11 \mathrm{~m}$ de la façade nord du SW 1.

16 La zone du SW 1 a livré un lot important de mobilier céramique dans les couches d'abandon et les niveaux de circulation. En attente d'une étude céramologique, il est encore difficile d'établir un phasage précis. Le dégagement du vestibule d'entrée ouest a livré un lot de fragments de scellés, confirmant ainsi la destination administrative du bâtiment. En outre, il est à noter que le bâtiment a souffert d'un ou plusieurs incendies. La proportion remarquable d'éléments charbonneux sur l'ensemble des secteurs fouillés a permis une campagne de prélèvements systématiques qui seront proposés à l'analyse ${ }^{14} \mathrm{C}$.

L'opération de 2019 sur SW 1 a livré des résultats riches et inattendus. Les dégagements ont permis d'alimenter le dossier encore relativement maigre des structures installées en périphérie proche de la carrière $\mathrm{P}$. L'état de conservation des structures en place a permis une première appréciation des modalités de construction du bâtiment, de son plan général et de l'interaction avec son environnement proche. Le bâtiment s'étend vraisemblablement au-delà de la zone d'opération 2019 à l'est et au nord-est de celle-ci, ainsi que semble le suggérer le départ de murs de grande et moyenne section chainés au parement intérieur de l'enceinte nord (secteur V) et orientés vers l'est en direction de la zone d'éboulis. Au terme de cette opération initiale, il convient alors d'envisager la poursuite des opérations de dégagement sur ces deux secteurs en privilégiant l'hypothèse que le SW 1 constitue la partie orientale d'un complexe bâti de grande ampleur.

\section{Survey des structures du plateau de Hatnoub}

Dominique Farout, Sameh Michel

Une première campagne de prospection a été menée par D. Farout, M. Crépy et O. Lavigne dans la zone de structures situées au nord-ouest de la carrière $\mathrm{P}$ pour en compléter la carte.

En raison d'un projet avancé de construction d'une route devant traverser le plateau de Hatnoub, D. Farout et S. Michel ont procédé à une prospection préventive de sauvetage sur le tracé balisé de cette nouvelle route. Assez peu de structures antiques se trouvent sur ce tracé proprement dit. En revanche, dans le ouadi qui commence immédiatement à l'ouest du nouveau tracé et au sud de la voie antique, et qui s'étend jusqu'au Kobri Sidi Abd el-Samad, des structures d'habitat ont été découvertes sur la pente faisant face au sud ainsi que des aménagements pour gérer les eaux de ruissellement. Ce ouadi a ainsi été nommé "Challalat» (Cascades). Un premier examen des céramiques de surface permet de conclure à une présence depuis au plus tard l'Ancien Empire. Des prélèvements de céramiques ont été effectués pour étude. 
Fig. 7. Vue des installations dans le ouadi Challalat (D. Farout).

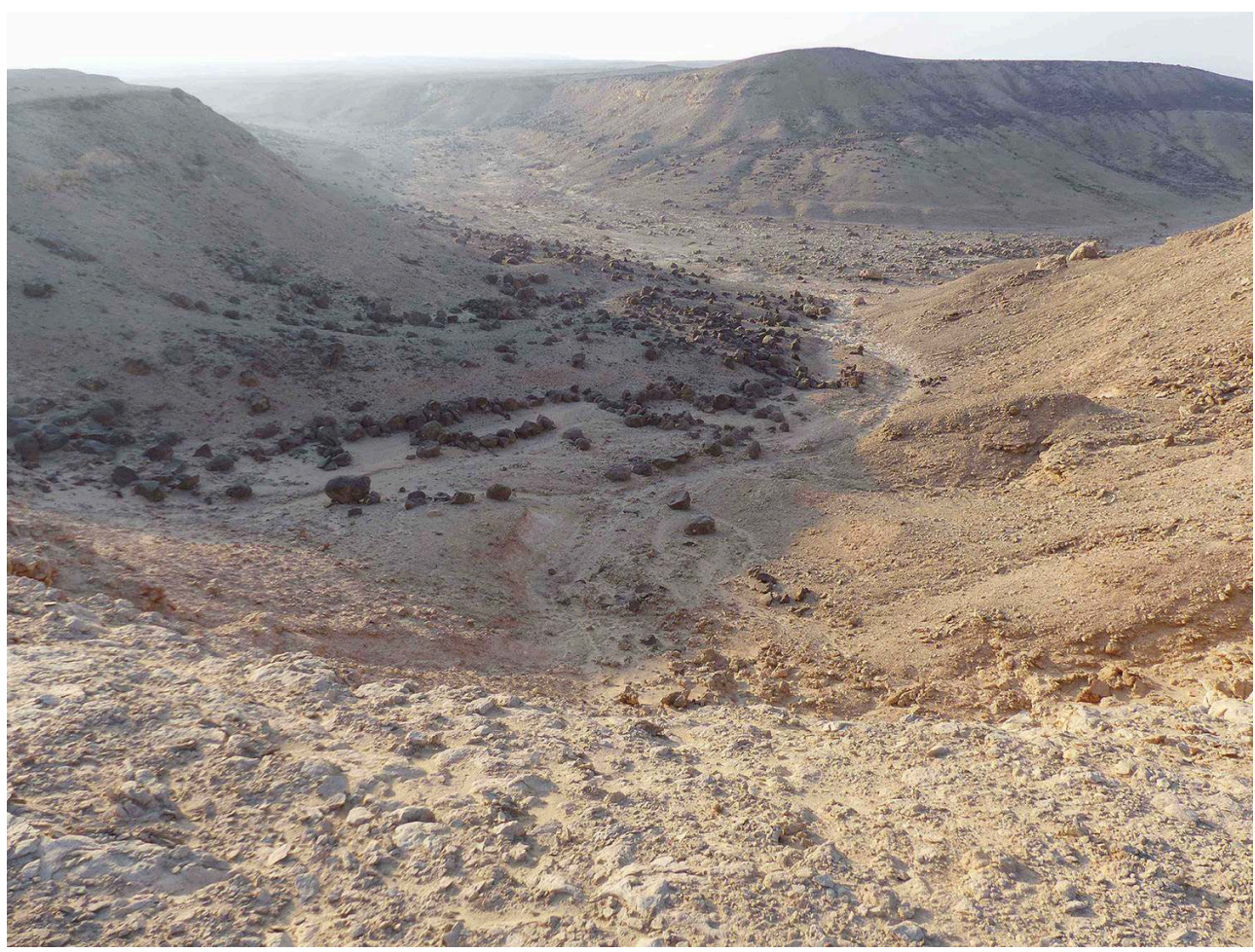

(C) Ifao. 17134_2019_NDMPF_006

\section{4. Étude géographique et géomorphologique aux alentours de la carrière $P$}

Maël Crépy, Yannis Gourdon

L'étude géographique et géomorphologique de la carrière $\mathrm{P}$ s'est déroulée du $7 \mathrm{au}$ 15 septembre 2019, dans les environs immédiats de la carrière $P$, dans la carrière ellemême et dans d'autres carrières de la région d'exploitation ancienne et/ou moderne (fig. 1). Grâce à des échanges permanents avec les autres membres de la mission, notamment Y. Gourdon, O. Lavigne et J. Fage, elle a permis des avancées significatives relatives à la problématique hydraulique ainsi qu'à la compréhension de la formation et de l'exploitation de la carrière $P$. 
Fig. 8. Itinéraires de prospection dans la zone de la carrière $P$ d'après une vue Google Earth (M. Crépy).

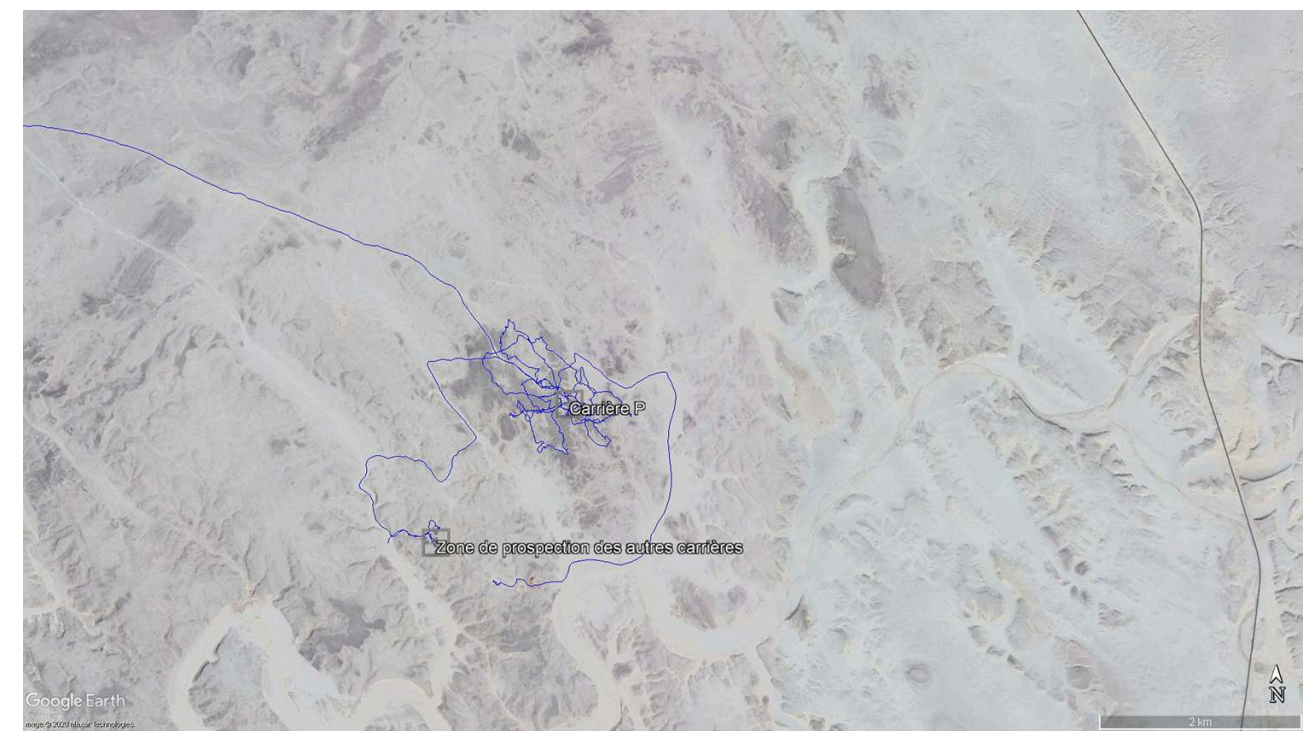

(C) Ifao. 17134_2019_NDMCN_001

\subsection{L'approvisionnement en eau}

L'étude a montré que la région du plateau calcaire de Hatnoub était extrêmement aride et qu'aucune ressource en eau exploitable n'est actuellement observable en surface ou à faible profondeur. Le plateau, très plat dans l'ensemble, mais assez accidenté dans le détail, est coupé par quelques grands et profonds oueds dont les lits sont en grande partie remplis de sables éoliens et d'alluvions anciens. De nombreux oueds secondaires traversent le sommet du plateau et en dissèquent plusieurs zones. La plupart d'entre eux ont un lit rocheux. Ils ne semblent pas avoir concentré de grandes quantités d'eau récemment : la présence dans le lit de ces oueds de vestiges non perturbés (fig. 2) avec des céramiques de l'Ancien Empire en surface confirme la rareté des précipitations durant l'Holocène récent, qui se sont avérées insuffisantes pour perturber l'organisation des cabanes. Bien que des traces de sources aient été observées sur le terrain, aucune d'entre elles n'a conservé de vestiges d'aménagement et d'exploitation. Il est donc très probable que leur assèchement ait précédé l'occupation de la région. Aucun vestige de puits, ou de creusement de puits, n'a été observé jusqu'à présent, et aucun bâtiment ne semble avoir été utilisé comme citerne. Il est donc très probable que l'approvisionnement en eau ait dû être assuré à partir de la seule vallée du Nil, bien qu'il ne soit pas totalement exclu qu'il y ait eu des puits dans le lit des principaux oueds (qui seraient maintenant comblés et masqués par les dynamiques éoliennes ou alluviales). Cette dernière conclusion doit cependant être tempérée à la lumière des aménagements découverts au ouadi Challalat (voir supra), après le départ de M. Crépy. 
Fig. 9. Cabanes antiques au milieu d'un oued (M. Crépy).

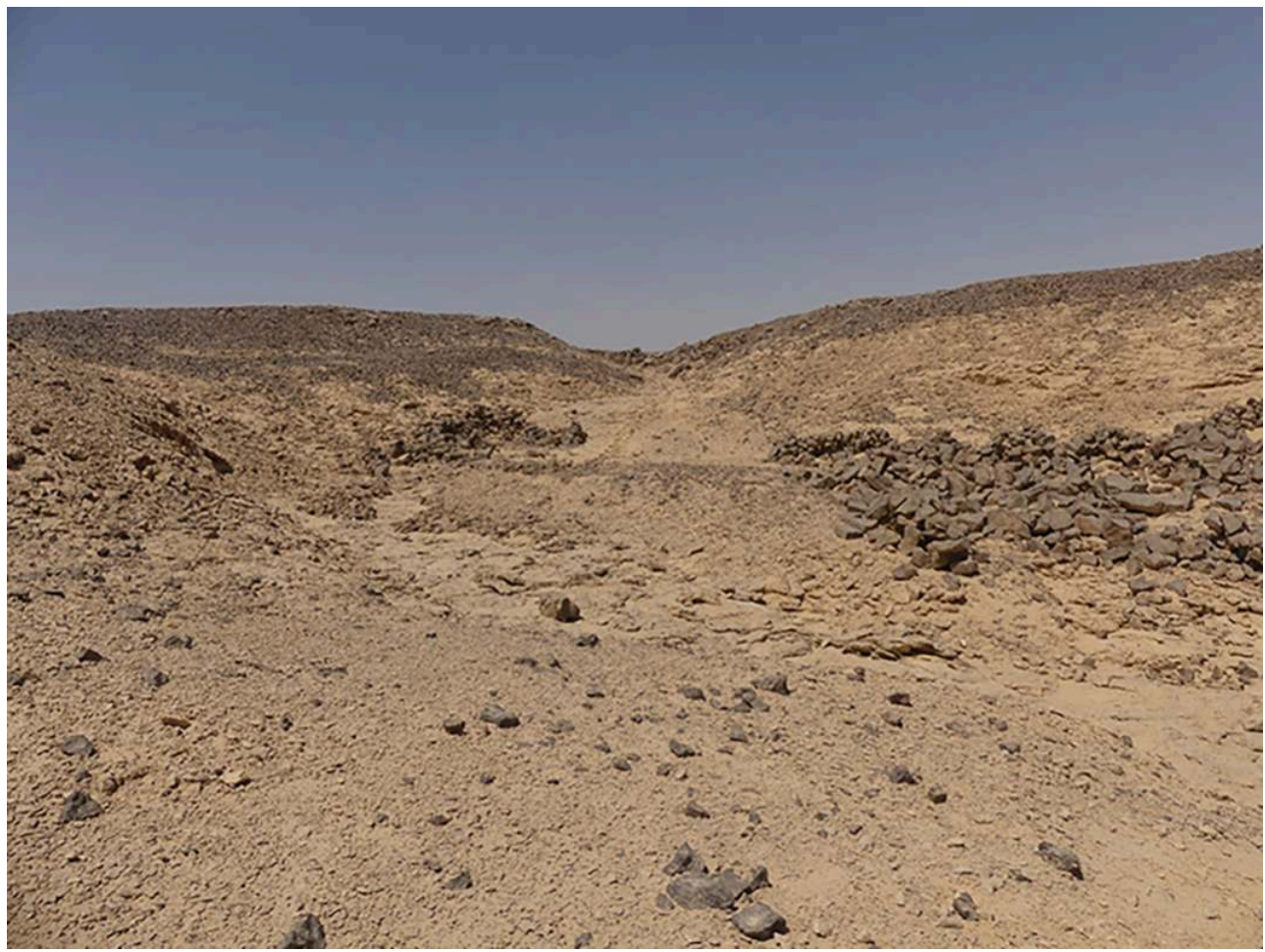

(C) Ifao. 17134_2019_NDMPF_007

\subsection{Identification et utilisation des calcaires et argiles locaux}

La quasi-totalité des roches observées sur le terrain, aussi bien en place que sous forme de galets ou de blocs, sont des calcaires de divers types. En particulier, outre la calcite exploitée dans l'Antiquité, on trouve des chailles (exploitées pour la fabrication d'outils), des amas pulvérulents de carbonate de calcium, des calcaires gris à nummulites, des calcaires fossilifères grenus et enfin un calcaire blanc neigeux étincelant à grain très fin contenant très peu de bioclastes et de fossiles largement exploités pour construire des bâtiments, pour produire une bonne part de l'outillage lithique, et même pour former la semelle de la voie antique reliant la carrière au Nil. C'est ce dernier calcaire, presque omniprésent, qui prend avec le temps la patine très sombre observée dans toute la région (fig. 3). Enfin, dans de nombreuses zones, on observe des concentrations importantes de terra rossa (argiles et limons de décarbonatation typiques des milieux karstiques), à la fois en position naturelle et remaniée par les activités humaines. Ces argiles peuvent avoir été utilisées à diverses fins (artisanat, carrière et/ou vraisemblablement lubrification de rampes) en plus d'être un excellent indicateur pour identifier l'emplacement des dépôts d'albâtre. À ce sujet, Ahmed Saad, un des chauffeurs de la mission qui a travaillé comme carrier dans le passé, nous a dit que "l'albâtre aime la terre rouge », exposant ainsi l'un des moyens employés par les carriers pour identifier les gisements. 
Fig. 10. Patine sombre sur les calcaires blancs. Le galet fraîchement cassé a été prélevé sur la voie antique (M. Crépy).

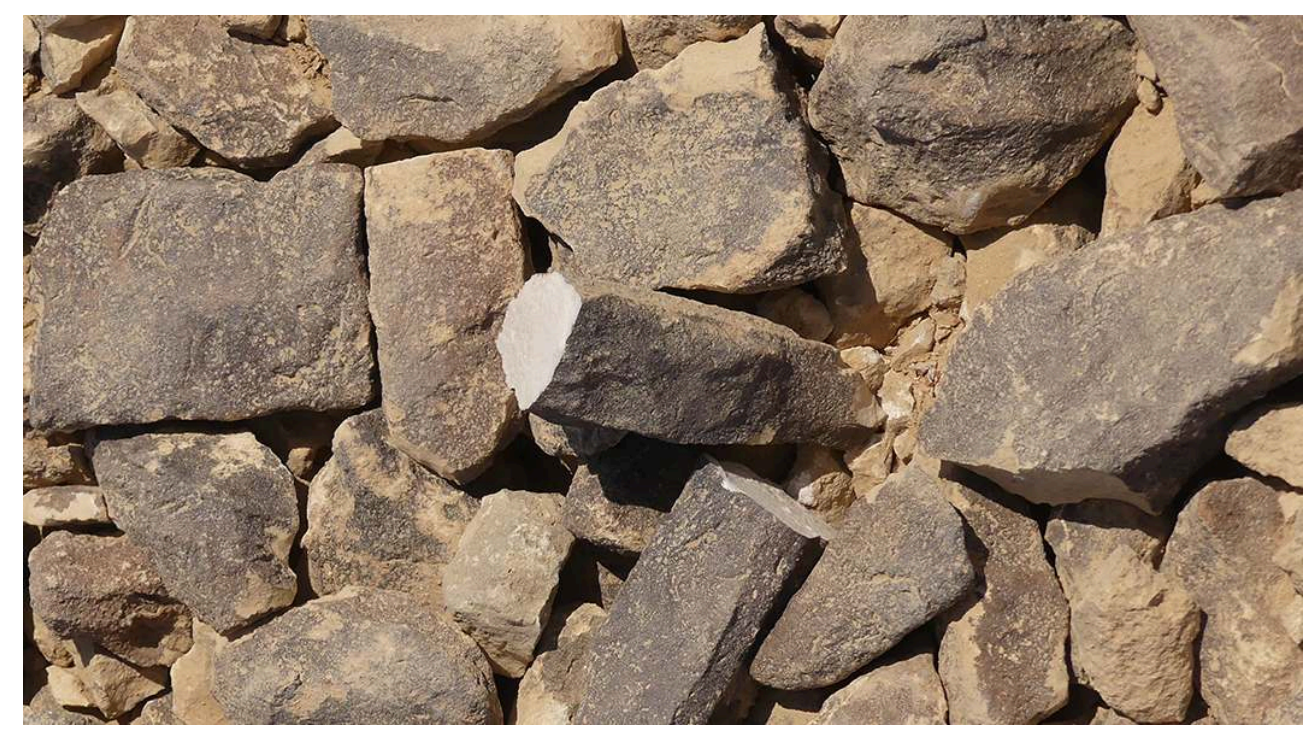

(c) Ifao. 17134_2019_NDMPF_008

\subsection{Formation et exploitation du gisement}

La carrière $\mathrm{P}$, dans son état de comblement partiel, a une profondeur maximale dépassant $25 \mathrm{~m}$. Elle se présente sous la forme d'un grand cirque relié à la surface topographique par une rampe incurvée. Sa profondeur totale pourrait être beaucoup plus importante, car au point le plus bas, on observe le sommet d'une excavation correspondant soit à une entrée de galerie, soit à une amorce de sape pour exploiter l'albâtre. D'autres entrées de galerie, partiellement masquées par le remblai, sont visibles à différentes hauteurs dans la face nord de la carrière. L'exploitation presque complète de la carrière $\mathrm{P}$ et son remplissage empêchent d'obtenir certaines réponses, principalement sur le type de gisement d'albâtre et sur sa morphologie ancienne, nous avons donc visité d'autres carrières. L'étude des carrières antiques et/ou modernes situées au sud-ouest de la carrière P montre que l'albâtre était principalement exploité dans des grottes naturelles, où il peut être trouvé dans des fractures de la roche comme remplissage karstique, ou dans des spéléothèmes tels que des stalagmites, des stalagtites ou des piliers.

On peut donc envisager, malgré la grande surface de la carrière $\mathrm{P}$, qu'elle constituait initialement une grotte remplie de spéléothèmes et de dépôts de calcite, similaire par exemple à la grotte du Ouadi Sannur, et qu'elle a été exploitée pour la première fois en sous-sol, en préservant le toit naturel de la grotte comme un ciel de carrière pendant une durée inconnue. Cette hypothèse fournirait une explication plausible quant à l'attribution du nom de Hatnoub, «le domaine de l'or ", à la carrière P. C'est dans cet environnement souterrain que la calcite sans patine ni érosion serait apparue, à la lumière des torches, très brillante et scintillante comme de l'or.

\section{Restauration des inscriptions}

Yannis Gourdon 

découvert une nouvelle inscription tracée à l'encre rouge. La mise en page de ce texte fragmentaire suggère qu'il s'agissait d'une inscription officielle introduite par le protocole royal qui a totalement disparu; seules quelques traces révèlent la présence d'une date peu lisible.

\section{Photogrammétrie}

Thomas Sagory, Benoît Touchard

Suite aux travaux de restauration des inscriptions, une nouvelle campagne photographique a été réalisée par T. Sagory et B. Touchard en vue d'un traitement de décorrélation dans le plugin DStretch et de la publication des inscriptions, ainsi que pour l'étude des objets notables issus des opérations archéologiques.

T. Sagory et B. Touchard ont également poursuivi les prises de vues, les vidéos documentaires et les relevés photogrammétriques sur le site de la carrière $P$ et aux alentours. Ces derniers visent à enrichir les données nécessaires à la génération de modèles en trois dimensions géo-référencés et multi-échelle de l'ensemble de la carrière et ses environs ainsi que des points d'intérêt sur les parois de la carrière (gravures, inscriptions peintes, traces d'outils, etc.). Les modèles ainsi générés permettent d'extraire divers types de documents destinés à la publication scientifique, la recherche et la valorisation.

De retour en France, les projets de photogrammétrie sont traités afin de produire :

- des modèles haute définition, une décimation de chaque modèle destinée à la publication ;

- des ortho-projections de chaque modèle en haute définition et une décimation en moyenne définition.

La production d'un modèle 3D global est en cours de réalisation. Ce modèle, qui regroupe l'ensemble des prises de vues pour les années 2016, 2017, 2018 et 2019, vise à répondre aux besoins de la recherche, à de multiples échelles.

\section{Conclusion}

31 Suite à cette saison 2019, notre compréhension du système de halage des blocs de la carrière $\mathrm{P}$ se confirme et se précise. La pente de la rampe elle-même, qui semble être constante sur toute sa longueur, avoisinerait $29 \%$. La mise au jour d'éléments se rapportant à des pièces d'échafaudages apporte de nouvelles informations sur l'organisation du travail et les réseaux de circulation à l'intérieur de la carrière. Toutes ces données confirment la nécessité de vider intégralement la descenderie, condition sine qua non pour produire une synthèse sur le fonctionnement du système de halage des blocs dans la carrière $P$.

Le dégagement du secteur SW 1 ouvre des perspectives très prometteuses pour les saisons à venir, notamment en termes de chronologie et d'organisation de l'espace et du travail autour de la carrière $\mathrm{P}$ et de son interaction avec celle-ci. 
prospections continuent de dévoiler de nouveaux sites sur le plateau de Hatnoub dont la datation préliminaire est en adéquation avec celle de la carrière $\mathrm{P}$. La première analyse géomorphologique du plateau a ouvert la voie à de nouvelles réflexions sur l'occupation du territoire; elle sera poursuivie et couplée à une enquête d'ethnogéomorphologie menée auprès des carriers modernes et notamment ceux qui ont travaillés dans la région de Hatnoub il y a quelques années, afin de mieux comprendre les méthodes de prospection et d'extraction de l'albâtre calcite.

Enfin, la mission de Hatnoub espère apporter une contribution notable à la compréhension de la chronologie de l'Ancien Empire, notamment dans le cadre du projet Meryt présenté par A. Quiles (Ifao) et sélectionné par l'ANR en juillet dernier.

\section{Diffusion et valorisation}

\subsection{Articles}

- Yannis Gourdon, « Hatnoub », in Laurent Coulon, Mélanie Cressent (éd.), Archéologie française en Égypte. Recherche, coopération, innovation, BiGen 59, Le Caire, Ifao, 2019, p. 148-153.

- Hannah Pethen, "Alabaster: The Golden Light of Eternity ", in Sinead Madden (éd.), Alabaster: Catalogue of the Exhibition at Ordovas Art Gallery, 20 September - 15 December 2018, Londres, Ordovas, 2018, p. 6-19.

- Olivier Lavigne, «New Typology for Old \& Middle Kingdom Stone Tools: Studies in the Hatnub Quarries in Egypt », in Building Knowledge, Constructing Histories. Proceedings of the 6th International Congress on Construction History (6ICCH 2018), July 9-13, 2018, Brussels, Belgium, vol. 2, Londres, Taylor \& Francis, 2018, p. 857-862.

\subsection{Colloques internationaux}

- Yannis Gourdon, Roland Enmarch, «Further Discoveries at Hatnub Quarry P: The 2016-2018 Seasons of the Hatnub Project/ Mission de Hatnoub ", $12^{\mathrm{e}}$ congrès international des égyptologues, Le Caire, 3-8 novembre 2019.

- Yannis Gourdon, «Missions royales et missions privées : la place du commanditaire des expéditions à Hatnoub dans les inscriptions de la VI ${ }^{\mathrm{e}}$ dynastie », colloque « King and Officials in the Old Kingdom: Separation and Interaction ", Unige, Genève, 11-13 septembre 2019.

- Yannis Gourdon, « Des carrières au Nil : hallage des pierres et maillage territorial sur le plateau de Hatnoub», poster présenté à l'occasion de la Red Sea Conference IX «Networked Spaces: The Spatiality of Networks in the Red Sea and Western Indian Ocean », Lyon, 2-5 juillet 2019.

- Roland Enmarch, "Graffiti from the "Era of the Nomarchs" at Hatnub Quarry ", colloque « Clamour from the Past: Graffiti, Rock Inscriptions and Secondary Epigraphy from Ancient Egypt », Ifao, Le Caire, 15-17 juin 2019.

- Yannis Gourdon, "The Use of Egyptian Alabaster during the old Kingdom: Consistencies and Discrepancies between Written and Archaeological Material Related to Hatnoub Quarries », colloque « Clamour from the Past: Graffiti, Rock Inscriptions and Secondary Epigraphy from Ancient Egypt », Ifao, Le Caire, 15-17 juin 2019. 


\subsection{Communication et valorisation scientifique}

- Participation de l'équipe au documentaire de Gédéon Programmes tourné sur site, Le Monde de Khéops, $1^{\text {re }}$ diffusion le 17 février 2020 sur Planète+.

- Participation de Yannis Gourdon à l'émission jeunesse C'est toujours pas sorcier, saison 1, épisode 2, « Le mystère des Pyramides ", https://www.france.tv/enfants/neufdouze-ans/ctps/ctps-saison-1/1129737-le-mystere-des-pyramides.html

INDEX

Année de l'opération : 2019

lieux https://ark.frantiq.fr/ark:/26678/pcrtVaA82gFQn7

chronologie https://ark.frantiq.fr/ark:/26678/pcrtPezBqzEcKR

sujets https://ark.frantiq.fr/ark:/26678/pcrtSu7E2QhoXv, https://ark.frantiq.fr/ark:/26678/

pcrtXk6sdvTjnE, https://ark.frantiq.fr/ark:/26678/pcrtyJc250eY3m, https://ark.frantiq.fr/ark:/

26678/pcrt2hsBAwey2e, https://ark.frantiq.fr/ark:/26678/pcrtoPowqxFzs7, https://

ark.frantiq.fr/ark:/26678/pcrtDlzbGxWvTo

Thèmes : IFAO

\section{AUTEURS}

\section{YANNIS GOURDON}

Égyptologue, université Lumière Lyon 2

\section{ROLAND ENMARCH}

Égyptologue, University of Liverpool

OLIVIER LAVIGNE

Tailleur de pierre

JÉRÔME FAGE

Archéologue, musée de Saint-Romain-en-Gal

DOMINIQUE FAROUT

Égyptologue, École du Louvre, Institut Khéops

SAMEH MICHEL

Intendant du chantier Hatnoub

MAËL CRÉPY

Géomorphologue, CNRS, UMR 5189 HiSoMA 


\section{THOMAS SAGORY}

Photographe, ministère de la Culture

BENOÎT TOUCHARD

Photographe, Summum 3D 